\title{
Molecular and genetic characterization of the capsule biosynthesis locus of Streptococcus pneumoniae type 23F
}

\author{
Judy K. Morona, ${ }^{1}$ David C. Miller, ${ }^{1}$ Tracey J. Coffey, ${ }^{2}$ \\ Christofer J. Vindurampulle, ${ }^{1}$ Brian G. Spratt, ${ }^{2}$ Renato Morona ${ }^{3}$ \\ and James C. Paton ${ }^{1}$
}

\author{
Author for correspondence: James C. Paton. Tel: +6188204 6302. Fax: +61 882046051. \\ e-mail: patonj@wch.sa.gov.au
}

\footnotetext{
1 Molecular Microbiology Unit, Women's and Children's Hospital, North Adelaide, South Australia 5006, Australia

2 Wellcome Trust Centre for the Epidemiology of Infectious Disease, Department of Zoology, University of Oxford, Oxford OX1 3PS, UK

3 Department of Microbiology and Immunology, University of Adelaide, Adelaide, South Australia 5005, Australia
}

\begin{abstract}
The authors have previously reported the nucleotide sequence of the $5^{\prime}$ and $3^{\prime}$ portions of the Streptococcus pneumoniae type 23F capsular polysaccharide biosynthesis locus (cps23f) (from dexB to cps23fB and from cps23fL to aliA). These regions of cps23f were very similar to the sequence reported for cps19f, the capsule locus of S. pneumoniae type 19F. However, Southern hybridization analysis indicated that no other genes closely related to cps19f are present in the cps23f locus. In this study long-range PCR was used to amplify and clone the section of the $S$. pneumoniae type $23 F$ capsule locus between cps $23 \mathrm{fB}$ and cps23fL. This region is $13 \mathrm{~kb}$ in size and contains 12 new ORFs, designated Cps23fC-E, I, J, and $T-Z$. Functions are proposed for all of the protein products, including functional homologues of Cps19fC-E, Cps19fI and Cps19fJ. A biosynthetic pathway for type $23 \mathrm{~F}$ capsular polysaccharide is also proposed.
\end{abstract}

Keywords: Streptococcus pneumoniae, capsule locus, capsular polysaccharide biosynthesis

\section{INTRODUCTION}

Streptococcus pneumoniae (the pneumococcus) is an important cause of invasive disease in human populations throughout the world, resulting in high morbidity and mortality. Control of pneumococcal disease is being complicated by the increasing prevalence of antibiotic-resistant strains and the suboptimal clinical efficacy of existing vaccines. S. pneumoniae produces a polysaccharide capsule, which is essential for virulence, because it protects the pneumococcus from the nonspecific immune defences of the host during an infection (Austrian, 1981). All fresh isolates from patients with pneumococcal infection are encapsulated, and spontaneous non-encapsulated (rough) derivatives of such strains are almost completely avirulent.

There are now 90 recognized serotypes of $S$. pneumoniae (Henrichsen, 1995), each of which produces a structurally distinct capsular polysaccharide (CPS). CPS

Abbreviation: CPS, capsular polysaccharide.

The GenBank accession number for the nucleotide sequence reported in this paper is AF030373. production requires a complex pathway including synthesis of the component monosaccharides, activation of each to a nucleotide precursor, co-ordinated transfer of each sugar, in sequence, to the repeating oligosaccharide, and subsequent polymerization, export and attachment to the cell surface. The exception to this is type 3, which has a very simple CPS repeat unit consisting of glucose and glucuronic acid, and appears to be synthesized by a processive transferase (Arrecubieta et al., 1995; Dillard et al., 1995). Classical genetic studies by Austrian et al. (1959) demonstrated that the genes encoding CPS biosynthesis are closely linked on the $S$. pneumoniae chromosome. Recently, this has been confirmed by sequence analysis of complete capsule loci (cps) from S. pneumoniae serotypes 1, 3, 14, 19F and 19B (Arrecubieta et al., 1995; Dillard et al., 1995; Guidolin et al., 1994; Kolkman et al., 1997; Morona et al., 1997a, b; Muñoz et al., 1997). These studies demonstrated that, in each case, the cps loci are located between $\operatorname{dex} B$ and ali $A$ in the pneumococcal chromosome. Moreover, with the exception of type 3, they have a common organization, with type-specific genes located in the central portion of the locus flanked by genes common to multiple serotypes. Thus, transformation from one capsular serotype 


\author{
$\rightarrow 4)-\beta-D-G_{p a l}-(1 \rightarrow 4)-\beta-L-R h a_{p}-(1 \rightarrow 4)-\beta-D-G l c_{p}-(1 \rightarrow$ \\ 32 \\ $\uparrow \uparrow$ \\ Glyc-ol-2- $\mathrm{PO}_{4}^{-} 1$ \\ $\alpha-\mathrm{L}-\mathrm{Rha}_{p}$
}

Fig. 1. Biological repeat unit of pneumococcal type $23 \mathrm{~F}$ capsular polysaccharide. This is based on the chemical structure previously published by Richards \& Perry (1988), but reflecting the fact that D-Glc is the first sugar. D-Glc $c_{p}$ glucose; D-Gal galactose; $\mathrm{D}-\mathrm{Rha}_{p}$, rhamnose; Glyc-ol, glycerol; $\mathrm{PO}_{4}^{-}$, phosphate.

to another can be achieved by homologous recombination between conserved sequences either within or flanking the $c p s$ loci. In recent years, extensive evidence for natural switching of capsule type amongst populations of $S$. pneumoniae has been reported. In particular, a highly successful multidrug-resistant clone of S. pneumoniae type $23 \mathrm{~F}$, originally isolated in Spain, has switched capsule type from $23 \mathrm{~F}$ to types $19 \mathrm{~F}, 19 \mathrm{~A}, 14$, 9N and 3 (Coffey et al., 1991, 1998b; Barnes et al., 1995; Nesin et al., 1998). The repeat unit structure of type $23 \mathrm{~F}$ CPS (shown in Fig. 1) is quite distinct from those of the other serotypes (van Dam et al., 1990), and in the case of type $19 \mathrm{~F}$ variants, capsule switching would necessitate the acquisition of at least nine genes (cps19fC through $K$ ) which do not have homologues in type $23 \mathrm{~F}$ (Morona et al., 1997a). Recently, we analysed the $5^{\prime}$ and $3^{\prime}$ termini of the cps locus of the Spanish multi-resistant type 23F clone and the homologous regions from eight otherwise genetically indistinguishable type $19 \mathrm{~F}$ variants, to identify recombinational junctions (Coffey et al., 1998a). In the present study, we have completed analysis of the entire $c p s 23 f$ locus from the Spanish multi-resistant type $23 \mathrm{~F}$ clone and propose a pathway for biosynthesis of type $23 \mathrm{~F}$ CPS.

\section{METHODS}

Bacterial strains and plasmids. The multiresistant $S$. pneumoniae type 23F Spanish strain SP-264 has been described previously (Coffey et al., 1998a). Pneumococci were routinely grown in Todd-Hewitt broth with $0.5 \%(\mathrm{w} / \mathrm{v})$ yeast extract or on blood agar. Escherichia coli K-12 DH5 $\alpha$ (Bethesda Research Laboratories) was grown in Luria-Bertani broth (Maniatis et al., 1982) with or without $1.5 \%(\mathrm{w} / \mathrm{v}$ ) Bacto-agar (Difco). Where appropriate, ampicillin was added to the growth medium at a concentration of $50 \mu \mathrm{g} \mathrm{m} \mathrm{m}^{-1}$. The vector pBluescript KS was obtained from Stratagene.

Bacterial transformation. Transformation of E. coli with plasmid DNA was carried out as described by Brown et al. (1979).

DNA manipulations. S. pneumoniae chromosomal DNA used in Southern hybridization experiments was extracted and purified using the Wizard Genomic DNA purification kit from Promega. Chromosomal DNA was purified according to the manufacturer's instructions except that cell lysis was induced by the addition of $0.1 \%(\mathrm{w} / \mathrm{v})$ deoxycholate followed by incubation at $37^{\circ} \mathrm{C}$ for $10 \mathrm{~min}$. Plasmid DNA was isolated from E. coli by the alkaline lysis method (Morelle, 1989). Analysis of recombinant plasmids was carried out by digestion of DNA with one or more restriction enzymes under the conditions recommended by the supplier. Restricted DNA was electrophoresed in $0.8-1.5 \%(\mathrm{w} / \mathrm{v})$ agarose gels with a Tris/borate/EDTA (TBE) buffer system as described by Maniatis et al. (1982).

Long-range PCR. The Expand Long Template PCR System (Boehringer Mannheim) was used for long-range PCR according to the manufacturer's instructions and was performed in a Hybaid Touchdown Thermal Cycler. The long-range PCR programme used consisted of 25 cycles, after an initial incubation at $92{ }^{\circ} \mathrm{C}$ for 2 min to allow complete denaturation of the DNA template. The first 10 cycles consisted of denaturation at $92^{\circ} \mathrm{C}$ for $30 \mathrm{sec}$, annealing initially at $55^{\circ} \mathrm{C}$ for $1 \mathrm{~min}$, decreasing by $1{ }^{\circ} \mathrm{C}$ per cycle to $45^{\circ} \mathrm{C}$ at cycle 10 , and elongation at $68^{\circ} \mathrm{C}$ for $15 \mathrm{~min}$. Cycles $11-25$ consisted of denaturation at $92^{\circ} \mathrm{C}$ for $30 \mathrm{~s}$, annealing at $55^{\circ} \mathrm{C}$ for $1 \mathrm{~min}$ at cycle 11 , decreasing to $45^{\circ} \mathrm{C}$ at cycle 25 , and elongation at $68^{\circ} \mathrm{C}$ for $15 \mathrm{~min}$ at cycle 11 , increasing by $1 \mathrm{~min}$ per cycle to $35 \mathrm{~min}$ at cycle 25 . The sequences of the two primers used are as follows: J39, homologous to the $3^{\prime}$ end of $C p s 19 f B$ (TAGTTCATGTAGTTGCAAGTGACATGCACAA, bases 2190-2220 of the cps $19 f$ sequence); and J27, complementary to $c p s 19 f \mathrm{~L}$ (GGCTGGATCCAAACCATCTGGACTT, bases $11787-11811$ of the $c p s 19 f$ sequence).

Southern hybridization analysis. Chromosomal DNA $(2.5 \mu \mathrm{g})$ was digested with appropriate restriction enzymes and the digests electrophoresed on agarose gels in TBE buffer. DNA was then transferred to a positively charged nylon membrane (Hybond- $\mathrm{N}^{+}$, Amersham) as described by Southern (1975), hybridized to digoxigenin (DIG)-labelled probe DNA, washed, and then developed using anti-DIG-alkaline phosphatase conjugate (Boehringer Mannheim) and 4-nitro blue tetrazolium/X-phosphate substrate according to the manufacturer's instructions. DIG-labelled lambda DNA, restricted with HindIII, was used as a DNA molecular size marker.

DNA sequencing and analysis. Nested deletions of pneumococcal DNA cloned into pBluescript KS were constructed by the method of Henikoff (1984) using the Erase-a-Base kit (Promega). This DNA was transformed into E. coli DH5 $\alpha$ and the resulting plasmid DNA was characterized by restriction analysis. Double-stranded template DNA for sequencing was prepared as recommended in the Applied Biosystems sequencing manual. The sequence of both strands was then determined using dye-labelled primers on an Applied Biosystems model 373A automated DNA sequencer. The sequence was analysed using DNASIS and PROSIS Version 7.0 software (Hitachi Software Engineering). The program BLASTX (Altschul et al., 1990) was used to translate DNA sequences and to conduct homology searches of the protein databases available at the National Center for Biotechnology Information, Bethesda, MD. Amino acid sequence alignments were performed with the program CLUSTAL w (Thompson $e t$ al., 1994). Hydropathy plots were generated according to the method of Kyte \& Doolittle (1982), and were aligned using PROFILEGRAPH (Hofmann \& Stöffel, 1989).

\section{RESULTS AND DISCUSSION}

\section{Isolation of the type 23F cps genes}

We have previously reported the nucleotide sequence for both the $5^{\prime}$ and the $3^{\prime}$ portions of the $S$. pneumoniae type 23F CPS biosynthesis locus (cps23f) (Coffey et al., 1998a). These regions of $c p s 23 f$ (from $\operatorname{dexB}$ to $c p s 23 f B$ and from $c p s 23 f L$ to aliA) were highly similar to the sequence reported for cps19f. However, Southern hybridization analysis indicated that homologues of the 


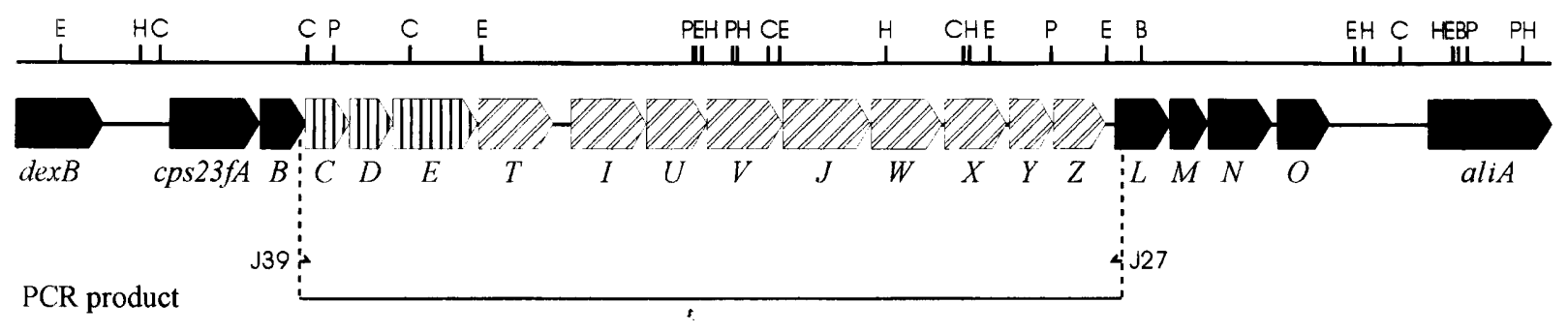

pJCP490
pJCP491
pJCP492
pJCP493
pJCP494
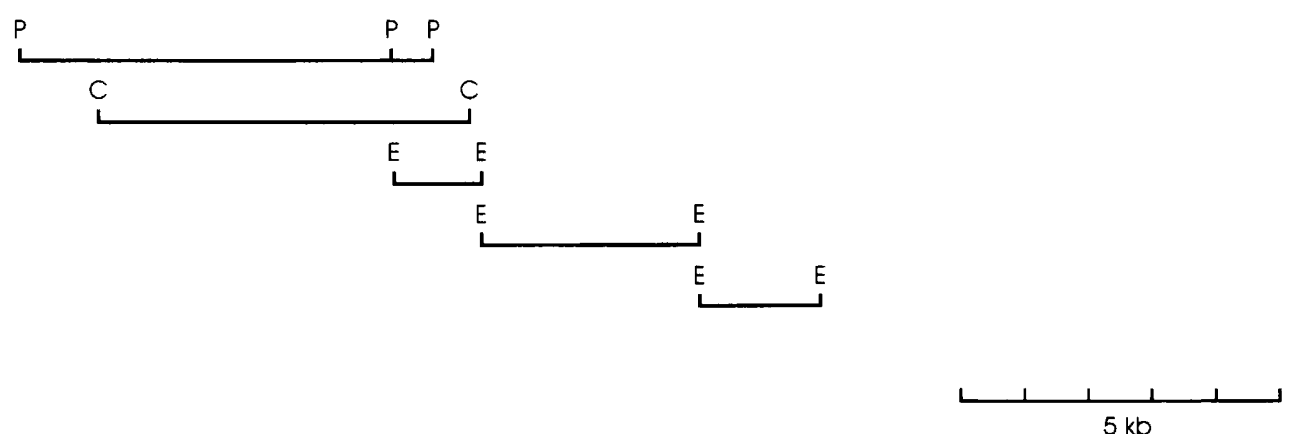

Fig. 2. Physical map of the S. pneumoniae type 23F CPS biosynthesis locus (cps23f). Black arrows represent previously decribed ORFs which are homologous to cps19f genes (Coffey et al., 1998a). Vertically hatched arrows represent cps23f ORFs with lesser, but nevertheless significant $(>70 \%)$ similarity to $c p s 19 f$ genes. Oblique-hatched arrows represent $c p s 23 f$ ORFs with negligible similarity to cps $19 f$ genes. Gene designations are indicated below the map; $\operatorname{cps} 23 f B-O$ are abbreviated to $B-O$, respectively. Restriction sites are as follows: B, BamHI; C, Clal; E, EcoRI; H, HindIII; P, Pstl. The longrange $P C R$ product and the regions of DNA subcloned into various recombinant plasmids are shown below the map.

remaining $c p s 19 f$ genes $(c p s 19 f C-K)$ were not present in the cps23f locus (Morona et al., 1997a). This central portion of $c p s 19 f$ would be replaced in the $c p s 23 f$ locus by the genes required specifically for type $23 \mathrm{~F}$ CPS biosynthesis. Long-range PCR was used to amplify this region of cps23f. The resultant PCR product was approximately $13 \mathrm{~kb}$ in size, approximately $4 \mathrm{~kb}$ larger than the equivalent region of $c p s 19 f$. A restriction map of the $13 \mathrm{~kb}$ PCR product was generated, using Bam HI, ClaI, HindIII, Pst I and EcoRI, and the PCR product was then subcloned, generating five plasmids with inserts as shown in Fig. 2.

Both strands of the pneumococcal DNA inserts of each of the above plasmids (or nested derivatives thereof) were subjected to sequence analysis in order to compile the sequence of the cps $23 f$ locus. The sequence across non-overlapping restriction sites and for both ends of the PCR product was obtained by direct dye-terminator sequencing of long-range PCR product using specifically designed primers. The compiled sequence was then linked to the existing $c p s 23 f$ data $(\operatorname{dex} B-c p s 23 f B$ and cps 23f $L$-aliA). As already reported (Coffey et al., 1998a), the first $4.6 \mathrm{~kb}$ at the $5^{\prime}$ end of the complete sequence has a high degree of similarity to the $c p s 19 f$ sequence. This region contained $\operatorname{dex} B$, the $5^{\prime}$ intergenic region (but it did not contain IS1202 as found in type 19F), and the homologues of cps19fA and $c p s 19 f B$ (cps23fA and $c p s 23 f B$, exhibiting $96.5 \%$ and $87.4 \%$ identity, respectively). A consensus $\sigma^{70}$ promoter sequence is located $5^{\prime}$ to $c p s 23 f A$. The promoter sequence is identical to that identified in cps loci from S. pneumoniae types 1,14 and
19F (Muñoz et al., 1997; Kolkman et al., 1997; Morona et al., 1997a), except for a single base difference in the -35 region (AAGACA for cps $23 f$, compared with TAGACA for the others). The next three genes in the cps23f locus (cps23fC, D and $E$ ) were also very similar to cps $19 f C-E(70 \cdot 3 \%, 74.3 \%$ and $72 \cdot 1 \%$ identity, respectively). The sequence then diverges suddenly at nucleotide 7460 of the cps $23 f$ sequence, which corresponds to the stop codon of $c p s 23 f E$. There are nine new potential open reading frames (ORFs) between $c p s 23 f E$ and $c p s 23 f L$; we have designated these $c p s 23 f T, I, U, V$, $J, W, X, Y$ and $Z$. Each ORF is preceded by a ribosomebinding site, and most ORFs are closely linked together, suggesting that they are transcriptionally coupled. The only significant intergenic gaps are 300 nucleotides between cps $23 f T$ and $c p s 23 f l$, and 160 nucleotides between cps $23 f Z$ and $c p s 23 f L$. However, no potential stemmed-loop structures were found in these regions. The $3^{\prime}$ end of the cps $23 f$ sequence again shows a high degree of similarity to the $c p s 19 f$ sequence, starting from nucleotide 17711. This is the first nucleotide of the cps $23 f \mathrm{~L}$ gene, which has $91.3 \%$ identity to $c p s 19 f \mathrm{~L}$. The remaining three genes of the $c p s 23 f$ locus $(c p s 23 f M, N$ and $O$ ) all exhibit greater than $97 \%$ identity to their respective cps19f homologues.

\section{Characterization of the cps23f genes}

The locations and several properties of $c p s 23 f C, D, E, T$, $I, U, V, J, W, X, Y$ and $Z$ are summarized in Table 1 . Significant similarities with other known proteins, 
Table 1. Summary of ORFs cps23fA-O

\begin{tabular}{|c|c|c|c|c|c|c|c|}
\hline ORF & $\begin{array}{l}\text { Location in } \\
\text { sequence }\end{array}$ & $\begin{array}{l}\text { Predicted } \\
\qquad M_{\mathrm{r}}\end{array}$ & $\begin{array}{l}\text { No. of } \\
\text { amino } \\
\text { acids }\end{array}$ & $\begin{array}{l}\text { Hydrophobicity } \\
\text { index }\end{array}$ & $\begin{array}{l}\text { Predicted } \\
\text { pI }\end{array}$ & $\begin{array}{c}\mathrm{G}+\mathrm{C} \\
\text { content } \\
(\mathrm{mol} \%) \dagger\end{array}$ & $\begin{array}{l}\text { Putative function of } \\
\text { predicted protein } \\
\text { product }\end{array}$ \\
\hline $\operatorname{cps} 23 f A$ & $2500-3945$ & 53516 & 482 & $0 \cdot 01$ & $8 \cdot 24$ & $38 \cdot 2$ & Regulatory protein \\
\hline $\operatorname{cps} 23 f B$ & $3947-4678$ & 28163 & 244 & -0.43 & $5 \cdot 72$ & $40 \cdot 7$ & Unknown \\
\hline $\operatorname{cps} 23 f \mathrm{C}$ & $4687-5379$ & 25450 & 230 & $0 \cdot 02$ & $9 \cdot 75$ & 42 & Chain length regulation \\
\hline $\operatorname{cps} 23 f D$ & $5389-6078$ & 24997 & 229 & $-0 \cdot 14$ & $8 \cdot 36$ & $38 \cdot 4$ & Chain length regulation \\
\hline $\operatorname{cps} 23 f E$ & $6093-7460$ & 52192 & 445 & $0 \cdot 16$ & $9 \cdot 42$ & $37 \cdot 4$ & $\begin{array}{l}\text { Glucose-1-phosphate } \\
\text { transferase }\end{array}$ \\
\hline $\operatorname{cps} 23 f T$ & $7491-8669$ & 45137 & 393 & $-0 \cdot 45$ & $5 \cdot 9$ & $36 \cdot 6$ & Rhamnosyltransferase \\
\hline $\operatorname{cps} 23 f I$ & $8969-10162$ & 45281 & 397 & $0 \cdot 86$ & $9 \cdot 99$ & $26 \cdot 7$ & Polysaccharide polymerase \\
\hline $\operatorname{cps} 23 f U$ & $10166-11137$ & 37476 & 323 & $-0 \cdot 21$ & $5 \cdot 08$ & 32 & Galactosyltransferase \\
\hline $\operatorname{cps} 23 \mathrm{~F}$ & $11144-12376$ & 47656 & 411 & $-0 \cdot 32$ & $9 \cdot 43$ & $30 \cdot 8$ & Rhamnosyltransferase \\
\hline $\operatorname{cps} 23 f J$ & $12378-13763$ & 51864 & 461 & $1 \cdot 00$ & $9 \cdot 41$ & $28 \cdot 4$ & Repeat unit transporter \\
\hline $\operatorname{cps} 23 f W$ & 13771-14919 & 44881 & 382 & -0.27 & 6.76 & $31 \cdot 2$ & Glycerophosphotransferase \\
\hline $\operatorname{cps} 23 f \mathrm{X}$ & $14955-15962$ & 36772 & 335 & -0.02 & $5 \cdot 74$ & $36 \cdot 6$ & $\begin{array}{l}\text { Glycerol-2-phosphate } \\
\text { dehydrogenase }\end{array}$ \\
\hline $\operatorname{cps} 23 f Y$ & $15999-16703$ & 26110 & 234 & 0.02 & $4 \cdot 56$ & $31 \cdot 9$ & $\begin{array}{l}\text { Glycerol-2-phosphate } \\
\text { cytidylyltransferase }\end{array}$ \\
\hline $\operatorname{cps} 23 f Z$ & $16718-17551$ & 31171 & 277 & $-0 \cdot 10$ & $4 \cdot 64$ & $32 \cdot 4$ & $\begin{array}{l}\text { Glyceraldehyde-2- } \\
\text { phosphotransferase }\end{array}$ \\
\hline $\operatorname{cps} 23 f L$ & $17711-18580$ & 32221 & 289 & -0.21 & $4 \cdot 7$ & 41 & $\begin{array}{l}\text { Glucose-1-phosphate } \\
\text { thymidylyltransferase }\end{array}$ \\
\hline$c p s 23 f M$ & $18581-19174$ & 22273 & 197 & -0.37 & $5 \cdot 05$ & $41 \cdot 2$ & $\begin{array}{l}\text { dTDP-4-keto-6-deoxy- } \\
\text { glucose-3,5-epimerase }\end{array}$ \\
\hline$c p s 23 f N$ & $19187-20236$ & 39041 & 349 & -0.46 & $5 \cdot 16$ & $41 \cdot 8$ & $\begin{array}{l}\text { dTDP-glucose-4,6- } \\
\text { dehydratase }\end{array}$ \\
\hline $\operatorname{cps} 23 f O$ & $20302-21153$ & 32387 & 283 & -0.52 & $4 \cdot 72$ & $41 \cdot 1$ & dTDP-L-rhamnose synthase \\
\hline
\end{tabular}

*According to Kyte \& Doolittle (1982), as implemented in Prosis. †Of coding region.

revealed by comparison with sequence databases, are described below.

The first five genes (ORFs) of the cps23f locus, $c p s 23 f A-E$, have a high degree of identity to $c p s 19 f A-E$; therefore the predicted functions of the gene products would be the same as described previously (Guidolin $e t$ al., 1994). Cps $23 f$ A (96\% identity to Cps19fA) may have a regulatory role, based on its similarity to the transcriptional regulator, LytR, from Bacillus subtilis (Lazarevic et al., 1992). The function of Cps $23 \mathrm{fB}(88.5 \%$ identity to Cps19fB) is unknown; however, homologues of this protein have, so far, only been identified in other Gram-positive CPS loci. Cps23fC and D $(69.6 \%$ and $79.3 \%$ identity to Cps19fC and D, respectively) are both predicted to be involved in chain length regulation and export of the CPS, based on their similarity to the first and last thirds of ExoP from Rhizobium (Sinorhizobium) meliloti (Glucksmann et al., 1993), respectively. Cps $23 \mathrm{fE}(71 \cdot 2 \%$ identity to Cps19fE) is likely to have the same function as the closely related Cps14E (95.8\% identity to Cps19fE), which transfers glucose to the lipid carrier (Kolkman et al., 1996).

The $c p s 23 f T$ gene encodes a putative protein which has limited similarity to putative mannosyltransferases from Aquifex aeolicus (18.9\% identity) (Deckert et al., 1998),
Synechocystis sp. (22.3\% identity) (Kaneko et al., 1995) and Archaeoglobus fulgidus (25.5\% identity) (GenBank accession number AE001104). Therefore, Cps23fT is probably a glycosyltransferase involved in the synthesis of the type $23 \mathrm{~F}$ CPS repeat unit. However, mannose is not present in the type $23 \mathrm{~F}$ capsule, and when the predicted specificities for the other glycosyltransferases present in the $c p s 23 f$ locus are considered, it seems most likely that Cps23fT is a rhamnosyltransferase.

Database searches with Cps23fl failed to identify any significant similarity to any other proteins. However, Cps23fI is a very hydrophobic protein and its hydropathy profile indicates that it has at least 12 hydrophobic, potentially membrane-spanning domains. This is a typical hydropathy profile for $\mathrm{Rfc}$-like proteins, which polymerize polysaccharide repeat units. Fig. 3 shows an alignment of the hydropathy profile for Cps23fI with those for putative polysaccharide polymerases from S. pneumoniae types 1,14,19B and 19F. In spite of negligible sequence similarities amongst these proteins, the profiles are very similar and on this basis, we suggest that Cps23fI is likely to be the polysaccharide polymerase for type $23 \mathrm{~F}$ CPS.

The cps23fU gene encodes a protein with similarity to Cps14J from S. pneumoniae type 14 ( $33.5 \%$ identity), an 


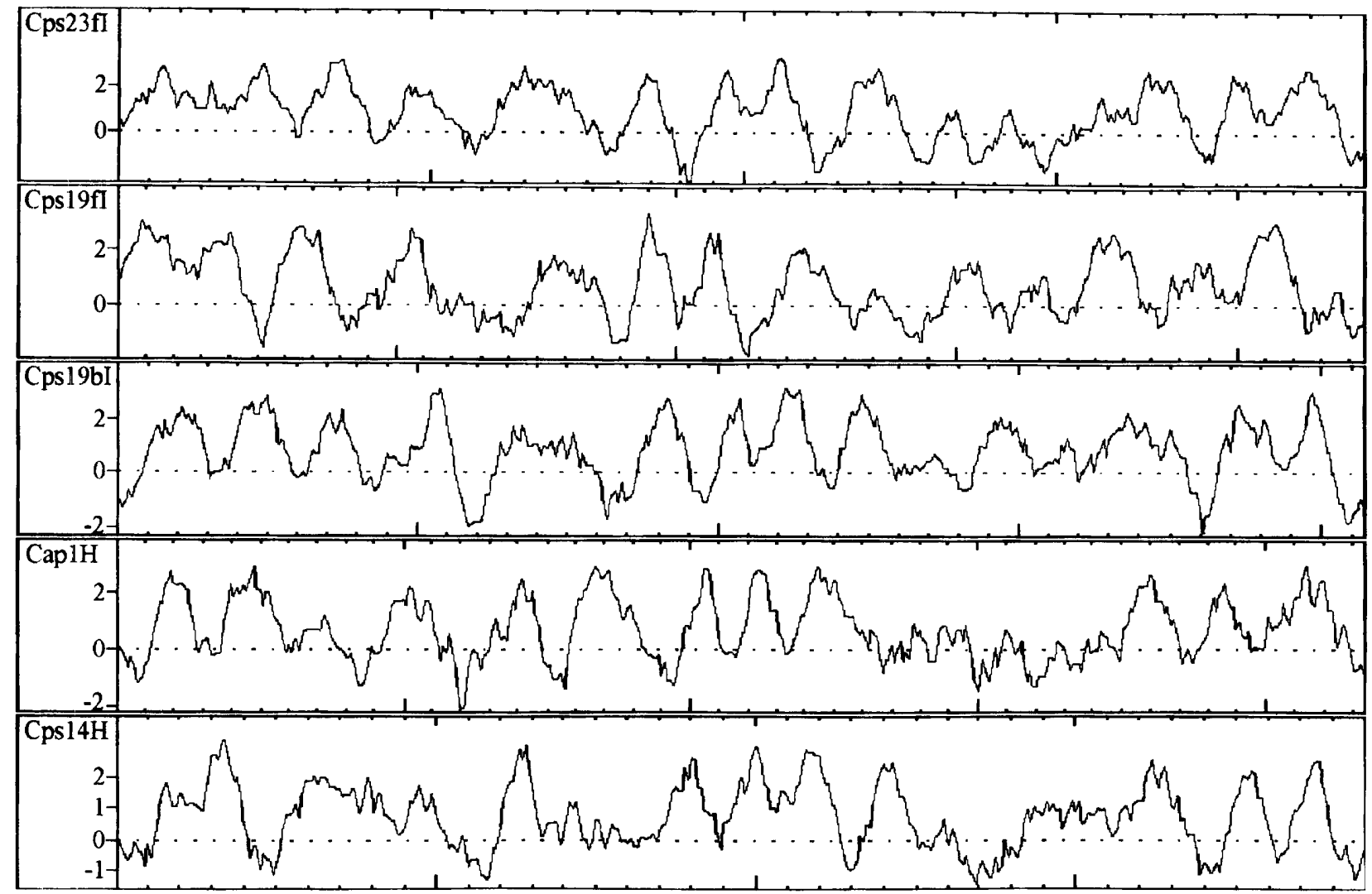

Fig. 3. Alignment of hydropathy plots of putative pneumococcal polysaccharide polymerases. Hydropathy plots for Cps23fl, Cps19fl from type 19F (Morona et al., 1997a), Cps19bl from type 19B (Morona et al., 1997b), Cap1H from type 1 (Muñoz et al., 1997) and Cps14H from type 14 (Kolkman et al., 1997) were generated by the method of Kyte \& Doolittle (1982) and aligned using PROFILEGRAPH (Hofmann \& Stöffel, 1989). Positive numbers on the $y$-axis indicate hydrophobic regions. The position of every 10 th amino acid is marked on each $x$-axis.

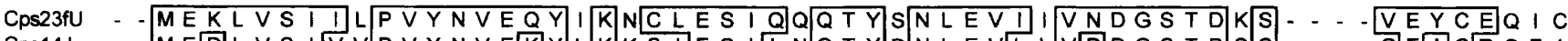

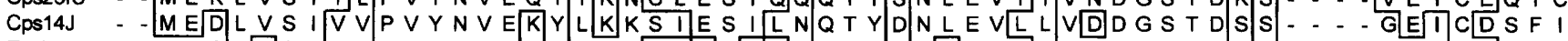

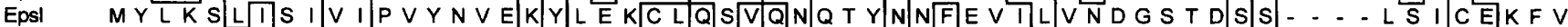

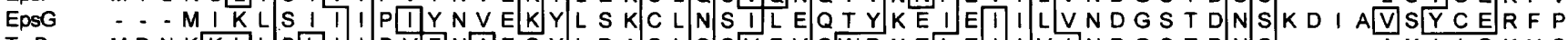

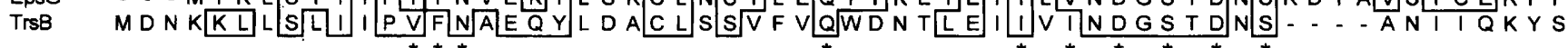

Cps23fU KI DSRFS I THKENGGLSDARNVG DDSKGDYLIFVDSDDFVDRDMVSYLVSCMENAEADI

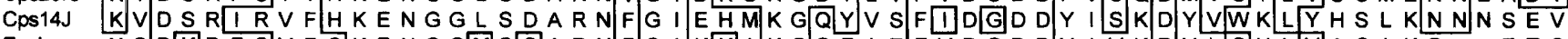

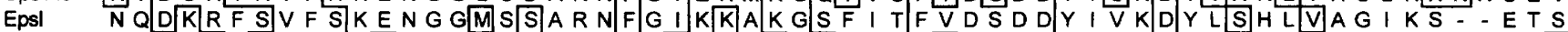

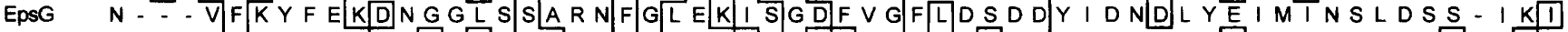

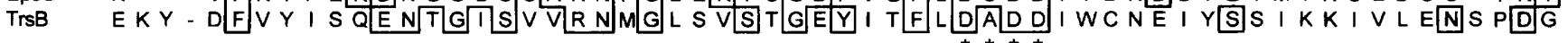

Fig. 4. Alignment of the $\mathrm{N}$-terminal portion (114 amino acids) of Cps23fU with Cps14J from S. pneumoniae type 14 (Kolkman et al., 1997), Epsl from Streptococcus thermophilus (Stingele et al., 1996), EpsG from Lactococcus lactis subsp. cremoris (van Kranenburg et al., 1997) and TrsB from Yersinia enterocolitica (Skurnik et al., 1995) using the default settings of the program CLUSTAL $W$ (Thompson et al., 1994). Identical residues are boxed; a hyphen (-) indicates absence of a residue. The residues marked by asterisks correspond to a motif previously found in a variety of rhamnosyl- and 6deoxyhexosyltransferases (Morona et al., 1995, 1997a).

experimentally determined galactosyltransferase (Kolkman et al., 1997). Cps23fU also has similarity to several other galactosyltransferases: EpsI from Streptococcus thermophilus (36\% identity) (Stingele et al., 1996), EpsG from Lactococcus lactis subsp. cremoris $(29 \cdot 6 \%$ identity) (van Kranenburg et al., 1997) and TrsB from
Yersinia enterocolitica (29.7\% identity) (Skurnik et al., 1995). The similarity is greatest for the first 100 amino acids at the amino-terminal end, and an alignment of this region is shown in Fig. 4. Interestingly, the most conserved regions are similar to potential active sites identified in a variety of Gram-negative rhamnosyl- 
and 6-deoxyhexosyltransferases (Morona et al., 1995). Thus, Cps23fU is likely to be the galactosyltransferase which adds galactose to the type $23 \mathrm{~F}$ CPS repeat unit.

The $c p s 23 f \mathrm{~V}$ gene encodes a putative protein which has no significant similarity to any other proteins on any database.

The $c p s 23 f J$ gene encodes a highly hydrophobic protein (Table 1) with similarity to several putative oligosaccharide repeat unit transporters: EpsM from Streptococcus thermophilus (32.1\% identity) (Stingele et al., 1996), Cps14L from S. pneumoniae (16.7\% identity) (Kolkman et al., 1997), TrsA from Yersinia enterocolitica (17.9\% identity) (Skurnik et al., 1995), and Cap8K protein of Staphylococcus aureus $(20.6 \%$ identity) (Sau et al., 1997). Cps23fJ has a typical hydropathy profile for this type of protein (result not shown), with 10-12 hydrophobic, membrane-spanning domains (Macpherson et al., 1995). Thus, Cps23fJ is likely to be the polysaccharide repeat unit transporter.

The cps $23 f W$ gene encodes a protein with $27.8 \%$ identity to the carboxy-terminal end of the TagF protein from $B$. subtilis, which is a glycerophosphotransferase (Pooley et al., 1992). It also has $37 \%$ identity to ORF1, which is located at the $3^{\prime}$ end of the cps14 locus (Kolkman et al., 1997). The type 23F CPS repeat unit has a glycerol phosphate side-chain attached to the Gal residue (Fig. 1 ), and so Cps $23 \mathrm{fW}$ is probably the transferase which adds glycerol-2-phosphate to the repeat unit.

The next three genes, $c p s 23 f X, Y$ and $Z$, encode putative proteins that we predict would be involved in the biosynthesis of CDP-2-glycerol, the precursor required for the addition of the glycerol 2-phosphate side-chain in type 23F CPS. Biosynthesis of CDP-2-glycerol probably occurs via a three-step pathway. The first step of this involves the synthesis of glyceraldehyde 2-phosphate from glyceraldehyde, which is catalysed by the kinase glyceraldehyde-2-phosphotransferase. The most likely candidate for this protein is Cps23fZ, which has similarity to NagD from E. coli ( $32 \%$ identity) (Plumbridge, 1989) and AraL from B. subtilis (26.3\% identity) (Sa-Nogueira et al., 1997); however, the functions of both NagD and AraL are unknown. These proteins also have similarity (25-30\% identity) to a group of proteins with 4-nitrophenylphosphatase activity; this reaction can proceed in either direction (depending on the physiological conditions), suggesting that these proteins may in fact function as kinases. Thus, Cps23fZ could be the enzyme glyceraldehyde-2-phosphotransferase. In the second step of the putative pathway, glycerol-2-phosphate dehydrogenase would catalyse the conversion of glyceraldehyde 2-phosphate to glycerol 2-phosphate. Cps23fX has similarity to glycerol-1-phosphate dehydrogenases from Archaeoglobus fulgidus $32.3 \%$ identity) (GenBank accession number AE000988), Methanococcus jannaschii ( $34.4 \%$ identity) (GenBank accession number U67518) and AraM from B. subtilis $(28.6 \%$ identity) (Sa-Nogueira et al., 1997). Thus, cps $23 \mathrm{f}$ probably encodes a glycerol-2-phosphate dehydrogenase. The final step involves the synthesis of
CDP-2-glycerol by the enzyme glycerol-2-phosphate cytidyltransferase. Cps23fy has $27 \cdot 5 \%$ identity to $\mathrm{RfbF}$ from Archaeoglobus fulgidus, a predicted glucose-1phosphate cytidylyltransferase (GenBank accession number AE001025). Thus, Cps23fY is likely to be the glycerol-2-phosphate cytidyltransferase, required for synthesis of CDP-2-glycerol.

The final four genes, cps $23 f L-O$, are almost identical to cps19fL-O, which have been shown to encode the four enzymes necessary for biosynthesis of dTDP-rhamnose, the precursor of rhamnose in the type 19F CPS repeat unit (Morona et al., 1997a).

\section{Specificity of the cps23f genes}

To examine the relationship between cps $23 f$ and encapsulation loci of other $S$. pneumoniae serotypes, individual $c p s 23 f$ genes were labelled with digoxigenin and used in Southern blots to probe (at high stringency) ClaI-restricted chromosomal DNA from representative pneumococci belonging to the following types/groups: $2,3,4,6 \mathrm{~A}, 6 \mathrm{~B}, 7 \mathrm{~F}, 8,9 \mathrm{~N}, 9 \mathrm{~V}, 12,14,16,17,18 \mathrm{C}, 19 \mathrm{~F}$, 19A, 19B, 19C, 22, 24, and two other 23F isolates. The genes $c p s 23 f A-E$ and $c p s 23 f L-O$, which have a high degree of identity to $c p s 19 f$ genes, were not used as probes; and the results can be inferred from those previously reported for cps19f (Morona et al., 1997a). The probes for cps23fl (bases 8819-10198), cps23fU (bases 10199-11061), cps23fV (bases 11062-12324), cps23fJ (bases 11063-13496), cps23fW (bases 1402815087), cps23fX (bases 15277-16063), cps23fY (bases 16064-16686) and cps23fZ (bases 16937-17572) hybridized only with the other type $23 \mathrm{~F}$ isolates. Unfortunately, isolates belonging to other members of serogroup 23 (types 23A and 23B) were not available for testing, so it was not possible to determine which of these group-23-specific genes are unique to type $23 \mathrm{~F}$. The $c p s 23 f T$ probe (bases 7526-8600) hybridized with DNA from serotypes $2,6 \mathrm{~A}, 6 \mathrm{~B}, 16,17,18 \mathrm{C}, 22$ and $23 \mathrm{~F}$. Interestingly, types 2,17 and 22 also contain $\beta(1 \rightarrow 4)$ linked rhamnose in their CPS repeat units, and the other reactive types also contain rhamnose in their CPS repeat units. Thus, the hybridization properties are consistent with the proposed function of the $c p s 23 f T$ gene product as a rhamnosyltransferase.

\section{Type 23F CPS biosynthetic pathway}

Database searches have enabled us to identify candidate functions for most of the ORFs in the cps $23 f$ locus. Three of the five transferases required for the synthesis of the repeat units have been assigned (Cps23fE, Cps23fU and Cps23f(W), as have the polysaccharide repeat unit transporter (Cps $23 \mathrm{fJ}$ ) and the polysaccharide polymerase (Cps23fI). The genes involved in the synthesis of both CDP-2-glycerol (Cps23fX-Z) and dTDPrhamnose (Cps23fL-O) have also been assigned. Cps23fC and Cps23fD are both believed to be involved in chain length regulation and export of the CPS, although this remains to be confirmed experimentally. However, the exact functions of Cps23fA and B are 


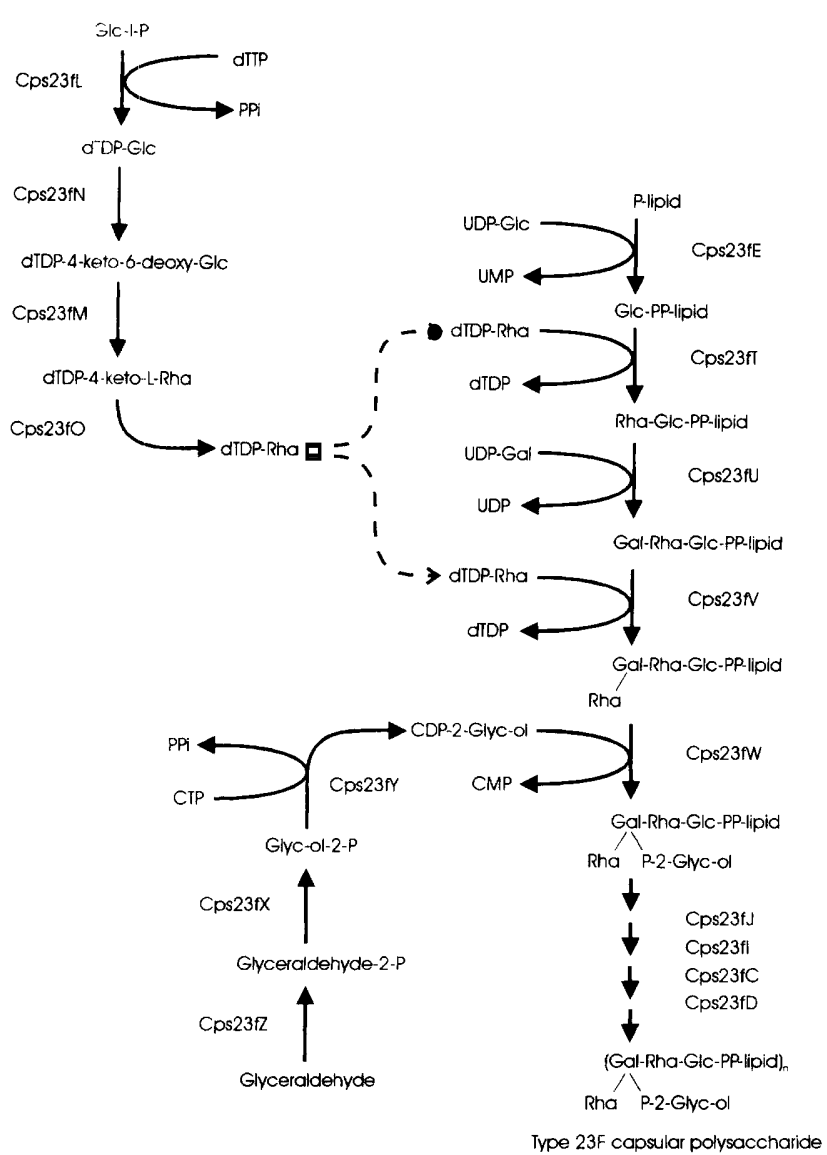

Fig. 5. Putative biosynthetic pathway for S. pneumoniae type 23F CPS.

unknown, although they are common to all pneumococcal CPS loci, except type 3 . Only two ORFs remain whose functions have not been clarified by comparison with other proteins of known function. Cps23fT does have some similarity to other transferases and is likely to be the rhamnosyltransferase which adds rhamnose to glucose via a $\beta(1 \rightarrow 4)$ linkage in the repeat unit backbone. This predicted function for Cps23fT is strengthened by the observation that the cps $23 f T$ specific probe hybridized to DNA from serotypes 2,17 and 22, which also contain $\beta(1 \rightarrow 4)$-linked rhamnose in their repeat units (van Dam et al., 1990). A second rhamnosyltransferase is required to add the rhamnose side-chain to the galactose in the backbone of type $23 \mathrm{~F}$ CPS, and the only ORF which could encode such a transferase is $c p s 23 f V$. The $\alpha(1 \rightarrow 2)$ linkage of this rhamnose side-chain to the galactose is quite unique and thus it is not surprising that $\mathrm{Cps} 23 \mathrm{fV}$ has no similarity to other known transferases whose sequences are available in GenBank. On the basis of the above information, we propose a biosynthetic pathway for $S$. pneumoniae type 23F CPS, as shown in Fig. 5.

\section{Conclusions}

In the present study we have characterized the typespecific portion of the cps locus of the $S$. pneumoniae type $23 \mathrm{~F}$ strain SP-264, a representative of the Spanish multidrug-resistant clone. This, combined with our previous study (Coffey et al., 1998a), indicates that cps $23 \mathrm{f}$ consists of 18 genes, located in the pneumococcal chromosome between $\operatorname{dex} B$ and aliA. The genes cps23fA-E, $I, J$ and $L-O$ have been named to indicate sequence similarity and/or functional homology with respective $c p s 19 f$ genes described previously (Guidolin et al., 1994; Morona et al., 1997a). The remaining genes (cps23fT-Z) have neither sequence similarity nor functional homology with $c p s 19 f$ genes. We have been able to assign putative functions to the majority of $c p s 23 f$ genes based on similarity with known proteins and with reference to the structure of the type $23 \mathrm{~F}$ repeat unit (Richards \& Perry, 1988). This enabled us to propose a biosynthetic pathway for type 23F CPS.

Whilst this manuscript was being revised, Ramirez \& Tomasz (1998) published the sequence of the cps locus from a Mexican multidrug-resistant clone of $S$. pneumoniae type $23 \mathrm{~F}$ (strain Him18). Comparison of the two $c p s 23 f$ sequences indicates a high degree of similarity for each of the ORFs $(97-100 \%$ identity at DNA level). However, the nomenclature used by Ramirez \& Tomasz (1998) for individual genes differs from that employed in the present study and in a previous description of the last four cps23f genes (Coffey et al., 1998a). The only notable difference within the two loci occurs in the gene we have designated $c p s 23 f X$; a single base change terminates the predicted product of this gene seven amino acids earlier in SP-264 than in Him18. Marked differences are also seen in the $5^{\prime}$ intergenic region, including a small deletion in SP-264 compared with Him18 and several other pneumococcal cps loci, affecting the first nucleotide of the -35 sequence. Ramirez \& Tomasz (1998) also found a truncated copy of IS1202 upstream of the cps23f promoter of Him18, which was absent in SP-264. Apart from the absence of IS1202, the $5^{\prime}$ intergenic region in SP-264 was very similar to the corresponding region from our previously described type 19F strain Rx1-19F (Guidolin et al., 1994; Morona et al., 1994). Interestingly, the $5^{\prime}$ intergenic region from Him 18 is virtually identical to the corresponding region from $S$. pneumoniae isolates belonging to types $19 \mathrm{~A}, 19 \mathrm{~B}$ and $19 \mathrm{C}$, sharing three separate DNA deletions between $\operatorname{dex} B$ and IS1202 (Morona, 1998). This is highly suggestive of a common clonal origin for these four strains. An additional difference between the two studies was noted in the pattern of hybridization using probes specific for cps $23 f T$. Using a 1074 bp probe spanning $91 \%$ of the cps23fT ORF, we detected hybridization to several other serotypes, including types 2 and $6 \mathrm{~B}$. These serotypes did not hybridize with the homologous probe in the study of Ramirez \& Tomasz (1998), presumably a consequence of differences in hybridization conditions or the length of the gene-specific probe employed.

The general organization of the cps23f locus is analogous to that reported for most other serotypes characterized to date (specifically types 1,14,19F and 19B) (Guidolin et al., 1994; Kolkman et al., 1997; Morona et al., 1997a, b; Muñoz et al., 1997). The 5' portion of these 
loci contains four genes (common to the majority of serotypes) believed to be involved in regulation and export of CPS. This is followed by a serotype-specific region encoding glycosyltransferases, the polysaccharide polymerase and the repeat unit transporter. The 3' region encodes enzymes for synthesis of activated monosaccharide precursors, some of which are common to several serotypes (e.g. those encoding dTDP-rhamnose synthesis). In $c p s 23 f$ the genes involved in biosynthesis of CDP-2-glycerol (cps $23 f X-Z)$ are also clustered in the $3^{\prime}$ portion immediately upstream of those for synthesis of dTDP-rhamnose. This conserved organization of $c p s$ loci undoubtedly facilitates genetic recombination which may result in switching of capsular type, or perhaps even generation of a novel functional cps locus encoding synthesis of a structurally distinct CPS.

\section{ACKNOWLEDGEMENTS}

This work was supported by grants from the National Health and Medical Research Council of Australia and from the Wellcome Trust (UK). B.G.S. is a Wellcome Trust Principal Research Fellow.

\section{REFERENCES}

Altschul, S. F., Gish, W., Miller, W., Myers, E. W. \& Lipman, D. J. (1990). Basic local alignment search tool. J Mol Biol 215, 403-410.

Arrecubieta, C., García, E. \& López, R. (1995). Sequence and transcriptional analysis of a DNA region involved in the production of capsular polysaccharide in Streptococcus pneumoniae type 3. Gene 167, 1-7.

Austrian, R. (1981). Some observations on the pneumococcus and on the current status of pneumococcal disease and its prevention. Rev Infect Dis 3 (Suppl.), S1-17.

Austrian, R., Bernheimer, H.P., Smith, E. E. B. \& Mills, G. T. (1959). Simultaneous production of two capsular polysaccharides by pneumococcus. II. The genetic and biochemical bases of binary capsulation. J Exp Med 110, 585-602.

Barnes, D. M., Whittier, S., Gilligan, P. H., Soares, S., Tomasz, A. \& Henderson, F. W. (1995). Transmission of multidrug-resistant serotype $23 \mathrm{~F}$ Streptococcus pneumoniae in group day care: evidence suggesting capsular transformation of the resistant strain in vivo. J Infect Dis 171, 890-896.

Brown, M. C. M., Weston, A., Saunders, J. R. \& Humphreys, G. O. (1979). Transformation of E. coli $\mathrm{C} 600$ by plasmid DNA at different phases of growth. FEMS Microbiol Lett 5, 219-222.

Coffey, T. J., Dowson, C. G., Daniels, M., Zhou, J., Martin, C., Spratt, B. G. \& Musser, J. M. (1991). Horizontal gene transfer of multiple penicillin-binding protein genes and capsular biosynthetic genes in natural populations of Streptococcus pneumoniae. Mol Microbiol 5, 2255-2260.

Coffey, T. J., Enright, M. C., Daniels, M., Morona, J. K., Morona, R., Hryniewicz, V., Paton, J. C. \& Spratt, B. G. (1998a). Recombinational exchanges at the capsular polysaccharide biosynthesis locus lead to frequent serotype changes among natural isolates of Streptococcus pneumoniae. Mol Microbiol 27, 73-83.

Coffey, T. J., Enright, M. C., Daniels, M., Wilkinson, P., Berron, S., Fenoll, A. \& Spratt, B. G. (1998b). Serotype 19A variants of the Spanish serotype 23F multiresistant clone of Streptococcus pneumoniae. Microb Drug Resist 4, 51-55. van Dam, J. E. G., Fleer, A. \& Snippe, H. (1990). Immunogenicity and immunochemistry of Streptococcus pneumoniae capsular polysaccharides. Antonie Leeuwenhoek 58, 1-47.

Deckert, G., Warren, P. V., Gaasterland, T. \& 12 other authors (1998). The complete genome of the hyperthermophilic bacterium Aquifex aeolicus. Nature 392, 353-358.

Dillard, J. P., Vandersea, M. W. \& Yother, J. (1995). Characterization of the cassette containing genes for type 3 capsular polysaccharide biosynthesis in Streptococcus pneumoniae. J Exp Med 181, 973-983.

Glucksmann, M. A., Reuber, T. L. \& Walker, G. C. (1993). Genes needed for the modification, polymerization, export, and processing of succinoglycan by Rhizobium meliloti: a model for succinoglycan biosynthesis. J Bacteriol 175, 7045-7055.

Guidolin, A., Morona, J. K., Morona, R., Hansman, D. \& Paton, J. C. (1994). Nucleotide sequence of an operon essential for capsular polysaccharide biosynthesis in Streptococcus pneumoniae type 19F. Infect Immun 62, 5384-5396.

Henikoff, S. (1984). Unidirectional digestion with exonuclease III creates targeted breakpoints for DNA sequencing. Gene 28, 351-359.

Henrichsen, J. (1995). Six newly recognized types of Streptococcus pneumoniae. J Clin Microbiol 33, 2759-2762.

Hofmann, K. \& Stöffel, W. (1989). PROFILEGRAPH: an interactive graphical tool for protein sequence analysis. Comput Appl Biosci 5, 151-153.

Kaneko, T., Tanaka, A., Sato, S., Kotani, H., Sazuka, T., Miyajima, N., Sugiura, M. \& Tabata, S. (1995). Sequence analysis of the genome of the unicellular cyanobacterium Synechocystis sp. strain PCC6803. I. Sequence features in the $1 \mathrm{Mb}$ region from map positions $64 \%$ to $92 \%$ of the genome. DNA Res 2, 153-166.

Kolkman, M. A. B., Morrison, D. A., van der Zeijst, B. A. M. \& Nuijten, P. J. M. (1996). The capsule polysaccharide synthesis locus of Streptococcus pneumoniae serotype 14: identification of the glycosyl transferase gene cps14E. J Bacteriol 178, 3736-3741.

Kolkman, M. A. B., Wakarchuk, W., Nuijten, P. J. M. \& van der Zeijst, B. A. M. (1997). Capsular polysaccharide synthesis in Streptococcus pneumoniae serotype 14: molecular analysis of the complete $c p s$ locus and identification of genes encoding glycosyltransferases required for biosynthesis of the tetrasaccharide subunit. Mol Microbiol 26, 197-208.

van Kranenburg, R., Marugg, J. D., van Swam, I. I., Willem, N. J. \& de Vos, W. M. (1997). Molecular characterization of the plasmid-encoded eps gene cluster essential for exopolysaccharide biosynthesis in Lactococcus lactis. Mol Microbiol 24, 387-397.

Kyte, J. \& Doolittle, R. F. (1982). A simple method for displaying the hydrophobic character of a protein. J Mol Biol 157, 105-132.

Lazarevic, V., Margot, P., Soldo, B. \& Karamata, D. (1992). Sequencing and analysis of the Bacillus subtilis lytRABC divergon: a regulatory unit encompassing the structural genes of the $N$-acetylmuramoyl-L-alanine amidase and its modifier. $J$ Gen Microbiol 138, 1949-1961.

Macpherson, D. F., Manning, P. A. \& Morona, R. (1995). Genetic analysis of the $r f b X$ gene of Shigella flexneri. Gene 155, 9-17.

Maniatis, T., Fritsch, E. F. \& Sambrook, J. (1982). Molecular Cloning: a Laboratory Manual. Cold Spring Harbor, NY: Cold Spring Harbor Laboratory.

Morelle, G. (1989). A plasmid extraction procedure on a miniprep scale. Focus 11.1, 7-8.

Morona, J. K. (1998). Characterization of the capsular polysaccharide biosynthesis loci of Streptococcus pneumoniae serogroup 19. PhD thesis, University of Adelaide. 
Morona, J. K., Guidolin, A., Morona, R., Hansman, D. \& Paton, J. C. (1994). Isolation, characterisation, and nucleotide sequence of IS1202, an insertion sequence of Streptococcus pneumoniae. $J$ Bacteriol 176, 4437-4443.

Morona, J. K., Morona, R. \& Paton, J. C. (1997a). Characterization of the locus encoding the Streptococcus pneumoniae type $19 \mathrm{~F}$ capsular polysaccharide biosynthetic pathway. Mol Microbiol 23, 751-763.

Morona, J. K., Morona, R. \& Paton, J. C. (1997b). Molecular and genetic characterization of the capsule biosynthesis locus of Streptococius pneumoniae type 19B. J Bacteriol 179, 4953-4958.

Morona, R., Macpherson, D. F., Van den Bosch, L., Carlin, N. I. A. \& Manning, P. A. (1995). Lipopolysaccharide with an altered Oantigen produced in Escherichia coli K-12 harbouring mutated, cloned Shigella flexneri rfb genes. Mol Microbiol 18, 209-223.

Muñoz, R., Mollerach, M., López, R. \& García, E. (1997). Molecular organization of the genes required for the synthesis of type 1 capsular polysaccharide of Streptococcus pneumoniae: formation of binary encapsulated pneumococci and identification of cryptic dTDP-rhamnose biosynthesis genes. Mol Microbiol 25, 79-92.

Nesin, M., Ramirez, M. \& Tomasz, A. (1998). Capsular transformation of a multidrug-resistant Streptococcus pneumoniae in vivo. J Infect Dis 177, 707-713.

Plumbridge, J. A. (1989). Sequence of the nagBACD operon in Escherichia coli $\mathrm{K} 12$ and pattern of transcription within the nag regulon. Mol Microbiol 3, 505-515.

Pooley, H. M., Abellan, F.-X. \& Karamata, D. (1992). CDPglycerol:poly(glycero-phosphate) glycerophosphotransferase, which is involved in the synthesis of the major wall teichoic acid in Bacillus subtilis 168, is encoded by tagF (rodC). J Bacteriol 174, 646-649.
Ramirez, M. \& Tomasz, A. (1998). Molecular characterization of the complete $23 \mathrm{~F}$ capsular polysaccharide locus of Streptococcus pneumoniae. J Bacteriol 180, 5273-5278.

Richards, J. C. \& Perry, M. B. (1988). Structure of the specific capsular polysaccharide of Streptococcus pneumoniae type $23 \mathrm{~F}$ (American type 23). Biochem Cell Biol 66, 758-771.

Sa-Nogueira, I., Nogueira, T. V., Soares, S. \& de Lencastre, H. (1997). The Bacillus subtilis L-arabinose (ara) operon: nucleotide sequence, genetic organization and expression. Microbiol 143, 957-969.

Sau, S., Sun, J. \& Lee, C. Y. (1997). Molecular characterization and transcriptional analysis of type 8 capsule genes in Staphylococcus aureus. J Bacteriol 179, 1614-1621.

Skurnik, M., Venho, R., Tolvanen, P. \& Al-Hendy, A. (1995). A novel locus of Yersinia enterocolitica serotype $\mathrm{O}: 3$ involved in lipopolysaccharide outer core biosynthesis. Mol Microbiol 17, 575-594.

Southern, E. (1975). Detection of specific sequences among DNA fragments separated by gel electrophoresis. J Mol Biol 98, 503-517.

Stingele, F., Neeser, J. R. \& Mollet, B. (1996). Identification and characterization of the eps (exopolysaccharide) gene cluster from Streptococcus thermophilus Sfi6. J Bacteriol 178, 1680-1690.

Thompson, J. D., Higgins, D. G. \& Gibson, T. J. (1994). CLuSTAL w : improving the sensitivity of progressive multiple sequence alignment through sequence weighting, position specific gap penalties and weight matrix choice. Nucleic Acids Res 22, 4673-4680.

Received 26 August 1998; revised 16 November 1998; accepted 11 January 1999. 